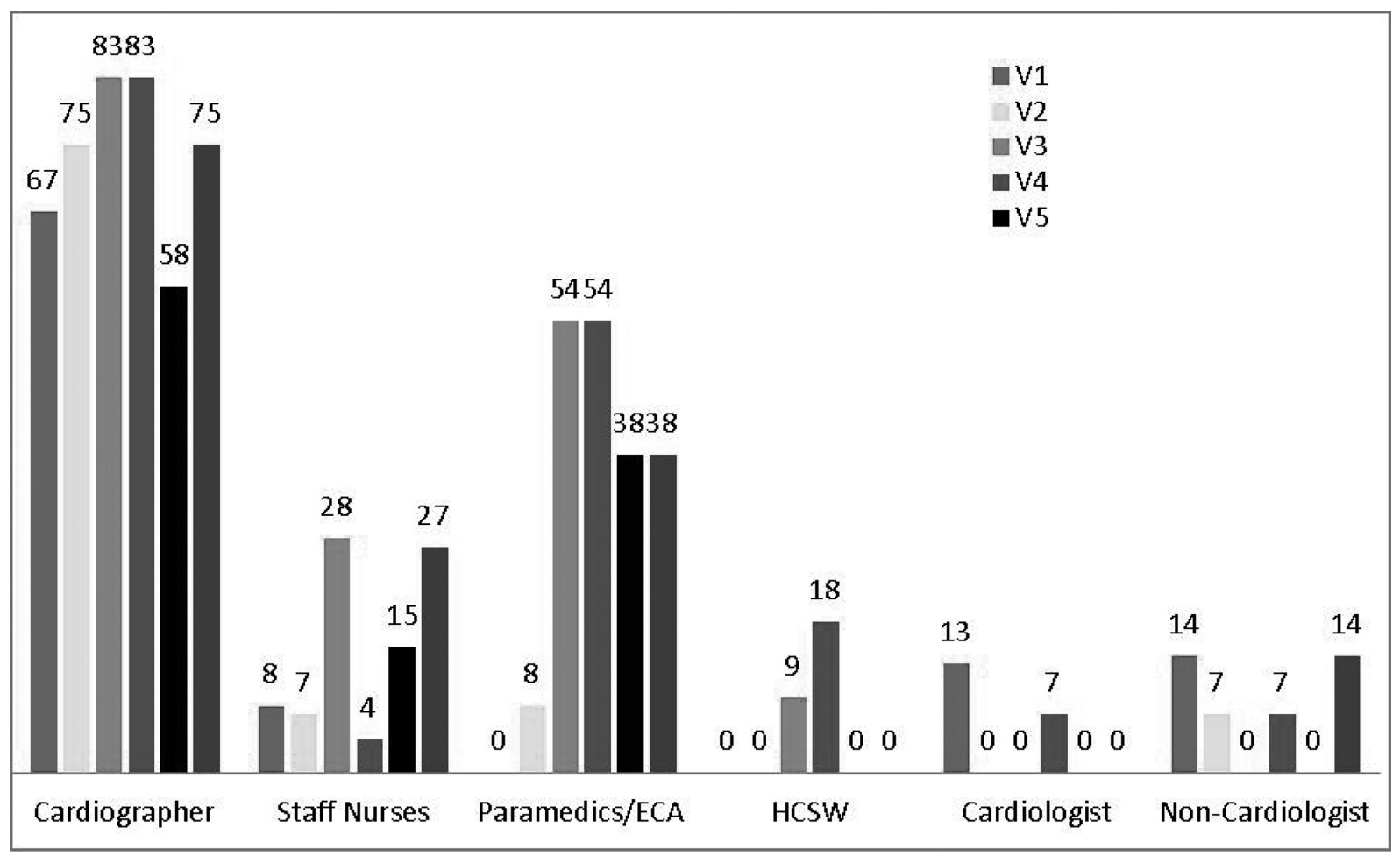

Abstract 58 Figure 2 Percentage of correctly placed electrodes according to professional group

Cardiac physiologists were the most accurate with a mean distance from the correct position across all leads of $6.7 \mathrm{~mm}$. Cardiographers performed similarly well with a mean distance of $6.9 \mathrm{~mm}$. Results for cardiology staff nurses, paramedics, non-cardiology physicians and non-cardiology staff nurses were $13.3,13.9,12.7$ and $17.7 \mathrm{~mm}$ respectively. Although healthcare support workers were the furthest away at $19.9 \mathrm{~mm}$, cardiologists were on average $17.2 \mathrm{~mm}$ away.

Secondary analysis compared all groups to cardiac physiologists. Figure 2 represents the percentage of correctly placed electrodes by each professional group. Although cardiographers performed well, cardiologists performed very poorly.

Conclusion These results demonstrate a significant lack of knowledge in the correct technique of ECG acquisition amongst healthcare staff outside of physiologists and cardiographers. Most notably, cardiology physicians appeared to be the least knowledgeable. This has the potential to result in artifactual changes in the ECG, particularly in respect of $\mathrm{R}$ wave progression, ST, and T wave morphology that could lead to misdiagnosis and administration of potentially harmful treatments. Further training is required and consideration of certification to ensure standardised practice.

\section{REMOTE PACEMAKER FOLLOW UP FROM A CONVENIENT COMMUNITY LOCATION - A PILOT STUDY}

Benedict Wiles, Hollie Cottrell*. University Hospital Southampton; *Presenting Author

\subsection{6/heartjnl-2016-309890.59}

Introduction All patients with a cardiac pacemaker require regular routine follow up. At an interval of no greater than twelve months battery longevity and lead parameters require assessment. This is usually undertaken in secondary care, by a trained cardiac physiologist, in a clinic setting. At University Hospital Southampton 2878 patients undergo routine pacemaker follow up. Pacemaker prevalence increases with patient age. Many patients with pacemakers are frail with multiple co-morbidities and a high percentage are reliant on hospital transport. Hospital attendance for this cohort can be challenging and costly. The pacemaker clinic has a high DNA (did not attend) rate. In 2012, 285 appointment slots were not utilised (around 15\% of total appointments).

Two-thirds of our pacemaker patients have a remote compatible Medtronic device. A single Medtronic carelink express monitor, placed in a convenient community location, would allow multiple patients to undergo remote monitoring. We hypothesise that this would increase patient satisfaction with pacemaker follow up, reduce wasted appointment time, minimise expenditure on hospital transport, decrease carbon footprint, and increase availability of on-site clinics and physiologists. We hypothesise that this would be a safe and effective method of conducting pacemaker follow up.

Method A Medtronic carelink express monitor was placed in a community health centre and a pilot group of 90 patients were invited to partake in community follow up. Patients were instructed on how to use the monitoring device and 57 patients were successfully enrolled. Patients were given an indication of when pacemaker follow up was required, but exact attendance time was entirely at their discretion. The carelink monitor was unmanned. Information was sent wirelessly to the hospital and assessed at a virtual follow up clinic. Results $100 \%$ of patients successfully utilised the remote device with a virtual DNA rate of $0 \%$. No patients required extra assistance in using the equipment at the time of download. The average time to assess a download was $6 \mathrm{~min}$, compared to $15 \mathrm{~min}$ for an on-site appointment. There were no 
adverse events during the follow up period. Average patient travel time and distance for follow up was reduced. Hospital transport was not required for any patient. The feedback from patients was positive and based around ease of travel, length of travel and stay, and flexibility to attend.

Conclusions and Implications This pilot study demonstrates the feasibility and benefits of remote pacemaker monitoring from a community location. This model could be replicated in the future by other Trusts.

\section{PATIENTS ADMITTED TO HOSPITAL WITH A DIAGNOSIS OF ATRIAL FIBRILLATION OUTSIDE OF STANDARD WEEKDAY WORKING HOURS AND AT WEEKENDS HAVE WORSE MORTALITY AND POORER SURVIVAL}

${ }^{1}$ Paul R Carter, ${ }^{2}$ Jennifer Reynolds, ${ }^{3}$ Hardeep Uppal, ${ }^{4}$ Suresh Chandran, ${ }^{5}$ Jaydeep Sarma, ${ }^{6}$ Rahul Potluri* ${ }^{1}$ Royal Free London NHS Foundation Trust; ${ }^{2}$ University of Birmingham; ${ }^{3}$ ACALM Study Unit in Collaboration with Aston Medical School; ${ }^{4}$ North Western Deanery; ${ }^{5}$ Deaprtment of Cardiology, University Hospital of South Manchester; ${ }^{6}$ ACALM Study Unit in Collaboration with Aston Medical School, Aston University; *Presenting Author

\subsection{6/heartjnl-2016-309890.60}

Introduction Atrial fibrillation (AF) is the most common cardiac arrhythmia and is associated with serious sequelae such as stroke and a significant mortality rate. Timely and optimal treatment of this condition can minimise these complications. There is evidence that patients with a range of conditions admitted out-of-hours (during evenings and weekends) have worse mortality compared to patient admitted during standard working hours. Mortality rates of AF patients admitted out of hours in the UK have not been previously been studied. We therefore aimed to study the impact of day and time of admission on short and long-term mortality in patients with AF.

Methods Anonymous data was collected from all patients with a diagnosis of AF admitted to several NHS hospitals in the

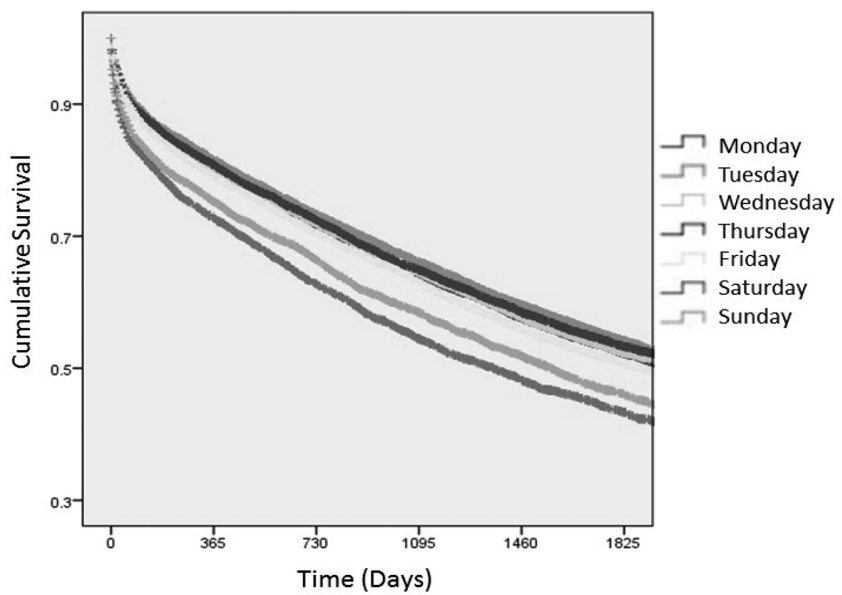

Abstract 60 Figure 1

North of England from $1^{\text {st }}$ January 2000 to $31^{\text {st }}$ March 2013 using the ACALM study protocol. The primary outcome of the study was "in and out of hospital mortality" within 30 days, 1 year, 5 years after the index admission.

Results 929,552 patients were admitted, of which 42,687 (4.6\%) patients had AF. 18,732 (43.9\%) of these were admitted during normal working hours (0900-1700; MondayFriday) and 23,955 (56.1\%) were admitted out of hours. Unadjusted crude mortality rates of patients admitted out of hours $(539 / 1000)$ were higher than those admitted during normal working hours (489/1000). Unadjusted crude death rates of patients admitted on Saturday (588/1000) and Sunday $(548 / 1000)$ were significantly higher than other days of the week (Figure 1). Cox regression analyses accounting for variations in age, gender, ethnic group and the ten most common causes of death in the UK (Listed in Table) showed that out of hours admission with AF conferred significantly worse

Abstract 60 Table 1 Demographics and comorbidities of atrial fibrillation patients

\begin{tabular}{|c|c|c|c|}
\hline & All patients & Admitted during normal hours(0900-1700 Monday to Friday) & Admitted outside of normal working hours \\
\hline Age, years $\pm S$. D & $74.2 \pm 12.5$ & $73.8 \pm 12.0$ & $74.5 \pm 12.9$ \\
\hline Male & $21891(51.3 \%)$ & $9881(52.7 \%)$ & $12010(50.1 \%)$ \\
\hline Female & $20796(48.7 \%)$ & $8851(47.3 \%)$ & $11054(49.9 \%)$ \\
\hline Ethnicity & $38052(89.1 \%)$ & $16900(90.2 \%)$ & $21152(88.3 \%)$ \\
\hline Caucasian & $735(1.7 \%)$ & $280(1.5 \%)$ & $455(1.9 \%)$ \\
\hline South Asian & $283(0.7 \%)$ & $118(0.6 \%)$ & $165(0.7 \%)$ \\
\hline Afro Caribbean & $107(0.3 \%)$ & $44(0.5 \%)$ & $63(0.3 \%)$ \\
\hline Oriental & $51(0.1 \%)$ & $21(0.1 \%)$ & $30(0.1 \%)$ \\
\hline Mixed & $3459(7.2 \%)$ & $1369(7.1 \%)$ & $2090(8.7 \%)$ \\
\hline \multicolumn{4}{|l|}{ Other } \\
\hline$I H D$ & $13900(32.6 \%)$ & 6214 (33.1\%) & $7686(32.1 \%)$ \\
\hline Heart Failure & $10992(25.8 \%)$ & $4599(24.6 \%)$ & $6393(26.7 \%)$ \\
\hline Lung Cancer & $926(2.2 \%)$ & $431(2.3 \%)$ & $495(2.1 \%)$ \\
\hline Breast Cancer & $810(1.9 \%)$ & $356(1.9 \%)$ & $454(1.9 \%)$ \\
\hline Colon Cancer & $340(0.8 \%)$ & $170(0.9 \%)$ & $170(0.7 \%)$ \\
\hline COPD & $6379(14.9 \%)$ & $2741(14.6 \%)$ & $3638(15.2 \%)$ \\
\hline Pneumonia & $5414(12.7 \%)$ & $2101(11.2 \%)$ & $3313(13.8 \%)$ \\
\hline CVD & 5007 (11.7\%) & $1934(10.3 \%)$ & $3073(12.8 \%)$ \\
\hline CKD & $4062(9.5 \%)$ & $1721(9.2 \%)$ & $2341(9.8 \%)$ \\
\hline Dementia & $3570(8.4 \%)$ & $1309(7.0 \%)$ & $2261(9.4 \%)$ \\
\hline
\end{tabular}

$\mathrm{IHD}=$ Ischaemic heart disease; $\mathrm{COPD}=$ Chronic Obstructive Pulmonary Disease; $C K D=$ Chronic Kidney Disease; CVD= Cerebrovascular Disease 